\title{
LE $14^{\circ}$ \\ COLLOQUE DE SALZBOURG SUR LA MÉCANIQUE DES ROCHES
}

\section{PAR \\ P. DUFFAUT ET P. LONDE *}

\section{Introduction}

Les 27 et 28 septembre 1963 s'est déroulé à Salz-

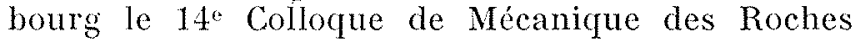
organisé et animé comme les précédents depuis 1951, par le docteur Mülclin. Pour la première fois, il s'agissait d'une «réunion régionale » de la nouvelle Société Internationale de Mécanique des Roches, fondée l'année précédente. La participation germanophone était largement prépondérante : un tiers d'Autrichiens, un tiers d'Allemands. Parmi vingt-cinq autres pays de toutes les parties du monde, on remarquait surtout les proches voisins, Suisse, Yougoslavie, Italie, mais aussi la Suède et le Royaume-Uni, nettement devant la France, l'Espagne, le Portugal et la Hollande. Les langues officielles étaient seulement l'anglais et l'allemand (avec traduction simultanée).

Sur les 270 inscrits, la grosse majorité est composée d'ingénieurs hydroélectriciens. Ensuite viennent des professeurs et des géologues, notamment de la République Fédérale. Les mineurs sont surtout allemands, anglais, et hollandais. Il est frappant de constater que la France n'était représentée que par six ingénieurs tous directement intéressés aux barrages.

L'éventail des 18 communications fait une place meilleure aux étrangers, puisqu'il y a seulement 5 allemands et 3 autrichiens en face de 3 yougoslaves, 2 anglais, 1 polonais, 1 hongrois, 1 portugais, 1 italien et 1 américain. Les professeurs y ont la majorité, mais les ingénieurs de génie civil apportent une contribution importante.

Les communications ne paraîtront que dans quelques mois dans la nouvelle revue Felsmechanik

* Electricité de France.

* * Coyne et Bellier.

(1) Les figures reproduites iei sont données sous toute réserve, sur la seule foi de notes prises en séance. und Ingenieurgeologie, qui a pris la suite de Geologie und Bamwesen.

L'exposé ci-dessous essaie d'analyser les contributions sonvent multiples des orateurs et de les rapprocher sous une forme synthétique. II s'attache davantage aux problèmes de tunnels et surtout de fondations de barrages.

\section{I. - Géologie appliquée: Description du massif rocheux}

\section{Krensch, New York (U.S.A.) :}

La figure 1(1) montre les quatre branches de la géologie appliquée aux Etats-Unis : mines, pétrole, hydrologie, génie civil et l'importance relative, dans

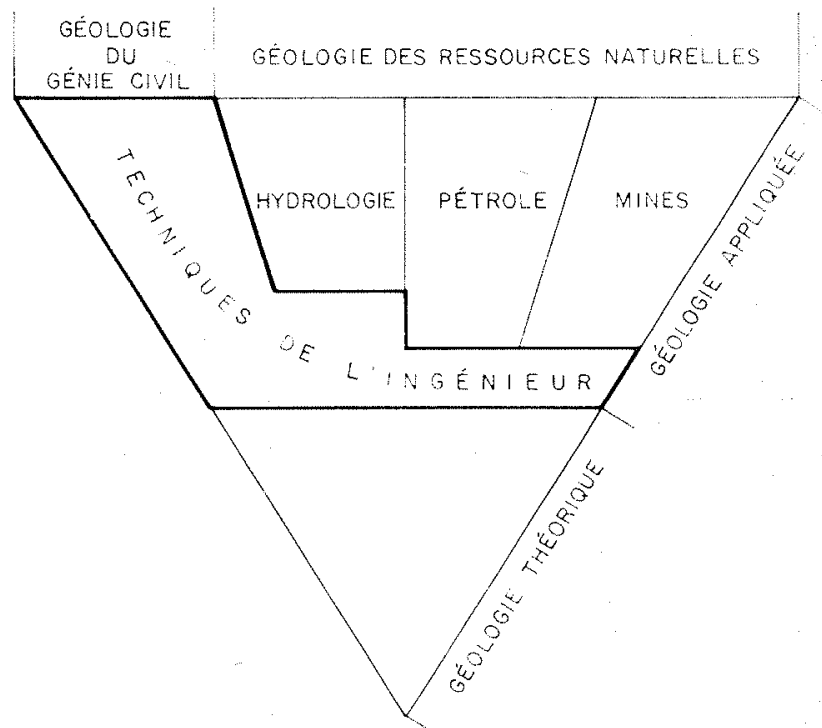

1/ Division de la géologie et importance des techniques de lingénieur en géologie appliquée. 


\section{P. DUFFAUT ET P. LONDE}

chaque branche, d'une part purement géologique, et d'une part qui nécessite la connaissance complète des techniques et des problèmes de l'ingénieur.

La principale difficulté pour le géologue est son adaptation à l'échelle de temps et à l'échelle dimensionnelle de l'ingénieur. L'étude détaillée des accidents (séismes, écroulements, rupture de fondations de barrages) est une base précieuse de la nouvelle science. C'est grâce à la mesure, introduite par les ingénieurs, que la géologie appliquée peut enfin passer du qualitatif au quantitatif.

Dörıng, Freiberg (Allemagne de l'Est).

La description naturaliste $\mathrm{du}$ massif rocheux doit s'attacher surtout aux structures. Le tableau I en donne une classification destinée à permettre un choix parmi les théories.

Horrmans, Koblentz (Allemagne Fédérale) :

Les renseignements fournis par les sondages ne prennent leur valeur que groupés sur des tableaux synoptiques comportant notamment la résistance de la roche, sa fragmentation, sa perméabilité et l'évolution du niveau piézométrique (l'auteur insiste sur les difficultés des essais d'eau classiques et attire l'attention sur les pertes de charge dans les tuyauteries).

Rocha, Lisbonne (Portugal):

Il faut distinguer avec soin les failles et les «joints» (2). La position individuelle de chaque faille joue un rôle et il faut vérifier la stabilité de chacun des blocs qu'elles isolent. Au contraire, la distribution des joints dans tout le massif modifie ses propriétés moyennes.

\section{II. - Considérations théoriques}

\section{a) Equilibre d'un pan de montagne}

WrTrke, Karlsruhe (Allemagne Fédérale) :

Sur un versant rocheux présentant une arête horizontale, deux discontinuités importantes isolent un tétraèdre monolithique (fig. 2) qui est supposé soumis seulement à son poids et à des réactions de frottement sur ses deux faces d'appui. La stabilité est discutée avec la notation vectorielle pour dif-

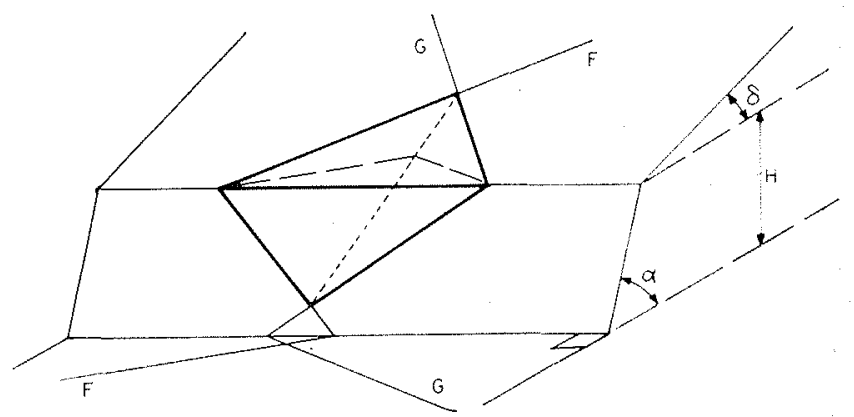

2/ Bloc découpé sur un versant par deux failles $F F$ et $G G$.

(2) Le mot français « joint » est d'emploi courant en maconnerie et en béton, il pourrait sans inconvénient être généralisé au massif rocheux (les géologues ne l'y emploient que pour la stratification).

\begin{tabular}{|c|c|}
\hline \multirow{2}{*}{$\begin{array}{l}\text { STRUCTURES } \\
\quad \text { NON } \\
\text { TECTONISÉES }\end{array}$} & 1 Sans discontinuités (roches non stratifiées)....... \\
\hline & 2 Discontinuités planes horizontales (stratification)... $\left\{\begin{array}{l}- \text { bancs cimentés } \\
- \text { bancs avec frottement } \\
- \text { bancs sans frottement }\end{array}\right.$ \\
\hline \multirow{3}{*}{$\begin{array}{l}\text { STRUCTURES } \\
\text { TECTONISÉES }\end{array}$} & 3 Discontinuités planes inclinées............. \\
\hline & 4 Discontinuités courbées (plis) $\ldots \ldots \ldots \ldots \ldots \ldots \ldots,\left\{\begin{array}{l}\text { - formes d'écoulement } \\
- \text { formes de cisaillement } \\
\text { formes de plissement }\end{array}\right.$ \\
\hline & 5 Discontinuités irrégulières. ............. \\
\hline
\end{tabular}


férents cas de figure. En particulier, on peut avoir glissement ou rotation du bloc. La méthode est évidemment généralisable à des blocs plus complexes que le tétraèdre, mais le calcul du coefficient de sécurité demande alors l'emploi de machines (3).

\section{b) Déformation et contraintes} dans les milieux continus

DörING :

Le massif rocheux peut être traité mathématiquement comme un milieu continu anisotrope, à condition de connaitre les propriétés mécaniques et physiques des matériaux remplissant les fissures. Le facteur temps est essentiel et le comportement du massif montre une viscosité qui l'apparente à un corps de Maxwell. Dans les cas particuliers simples et notamment lorsqu'il y a une seule direction de discontinuité (stratification ou schistosité), on peut arriver à un système d'équations d'équilibre. Hoeppener, Bonn (Allemagne Fédérale) :

Pour étudier les déformations affines d'un corps homogène, en grandeur relative et en direction, ainsi que les contraintes correspondantes, l'auteur utilise la projection plane de Lambert à partir d'une sphère unitaire (projection qui conserve le rapport des surfaces). Il peut ainsi comparer directement ses diagrammes à ceux de l'analyse structurale classique. L'étude des composantes vectorielles de la déformation en un point peut être ramenée à un petit nombre de diagrammes types.

La partie élastique des contraintes présente un tenseur symétrique, mais, s'il y a frottement, le tenseur est dissymétrique pour équilibrer le travail des forces extérieures. C'est toujours le cas dans les structures monoclinales.

Schenk, Giessen (Allemagne Fédérale) :

Dans le même esprit, les flux de déformation sont étudiés dans des couches plissées. De la conservation des flux, on peut attendre l'explication de nombreuses propriétés structurales, par exemple les différences entre une charnière synclinale et une voûte anticlinale.

Richter, Miskolc (Hongrie) :

La déformation, sous pression intérieure, d'une cavité cylindrique de section circulaire et de longueur 8 à 10 fois supérieure au rayon permet le calcul du module d'élasticité et du coefficient de Poisson. En étudiant deux saiggnées à $90^{\circ}$, on peut obtenir les contraintes principales et le rapport de la contrainte horizontale à la contrainte verticale dont le rôle a été souligné par Everling. On peut alors comparer la contrainte verticale avec la hauteur de couverture. L'auteur en déduit une relation théorique très générale donnant la déformation à partir de mesures très simples.

\section{c) Déformation aléatoire d'un milieu discontinu}

Litwiniszyn, Cracovie (Pologne):

Pour le soutirage d'un milieu pulvérulent con-

(3) Des calculs de ce genre ont été faits pour des cas concrets de bloc menaçant ruine, notamment en Norvège et pour le chantier du barrage du Dez en Iran. En France, pour l'étude du projet de barrage de Vouglans sur l'Ain, on a pu ajouter au poids propre la pression de la retenue future, des sous-pressions et la poussée transmise par un barrage. tenu dans une boìte, l'auteur avait déjà montré que, loin du trou, les surfaces horizontales se déformaient en donnant des courbes en cloche (lois de Gauss) (fig. 3). En reliant entre elle les probabilités de mouvement des grains situés dans trois niveaux

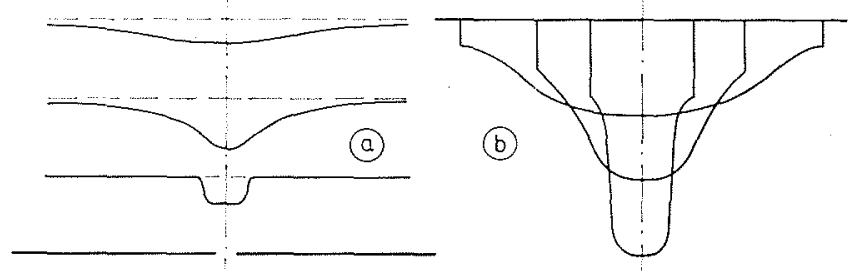

3/ a) Déformation des plans horizontaux dans un récipient rempli de sable et soutiré par un trou circulaire;

b) Discontinuités de la fonction qui définit la probabilité de mouvement vers le bas d'un grain de sable (échelle diffèrente).

superposés (ce qui revient à leur donner une certaine mémoire), il parvient à donner l'expression mathématique des discontinuités que l'on observe en se rapprochant du trou.

\section{d) Rupture des massifs rocheux}

Rocha :

Il faut séparer deux couples de paramètres $c$ et $\varphi$, les uns relatifs au matériau solide qui forme les blocs, les antres, aux matériaux contenus dans les fissures.

Comme il n'existe pas de théories satisfaisantes pour l'étude de la rupture, on est obligé de faire des mesures in situ et de compléter l'interprétation par des essais sur modèle. Dans tous les cas, in situ et sur modèle, le cisaillement doit être réparti dans un volume et non sur une surface.

Fumagalli, Bergame (Italie):

Contrairement à la cohésion, l'angle de frottement interne ne peut contribuer à la résistance qu'à partir du moment où il y a déplacement. Dans les ruptures fragiles, le frottement n'a pas le temps d'intervenir. C'est surtout le cas au voisinage de la surface libre, où l'une des contraintes principales est pratiquement nulle. Au contraire, dans les déformations sous triple étreinte, la cohésion joue un rôle négligeable devant les forces de frottement.

Lorsque la charge transmise à la fondation par un barrage augmente, le massif rocheux s'adapte aux efforts à la fois par des glissements relatifs et par des tassements. Ces deux types de mouvements ont des caractères visco-plastiques. Le béton à son tour s'adapte aux déformations des appuis, mais on doit penser qu'en général sa rupture interviendra avant celle du massif rocheux.

Dans la discussion finale, Krsmanovic a projeté deux figures tirées d'essais sur modèles qui illus. trent l'effet de l'étreinte sur le type de la rupture.

\section{II. - Mesures in situ}

\section{a) Dans la mine}

RoBErts et HAwres, Sheffield (Angleterre) :

L'emploi de la photoélasticité pour l'analyse des déformations, à la fois à la surface du massif et dans les forages, est répandu dans les mines de 


\section{P. DUFFAUT ET P. LONDE}

charbon d'Angleterre. Le disque employé est percé d'un trou central circulaire qui permet de concentrer les contraintes et augmente donc la précision des lectures. L'appareil placé en sondage comprend une source de lumière polarisée, une plaquette photoélastique, puis un dispositif optique et l'ensemble, très robuste, peut être scellé jusqu'à $5 \mathrm{~m}$ de profondeur.

Si ces appareils ont été tarés en laboratoire, dans un bloc de la même roche comprimé sous une presse, on peut mesurer les variations de contrainte et en particulier savoir si un seuil critique fixé à l'avance est atteint ou dépassé. (La contrainte absolue ne peut ètre mesurée que par carottage autour de l'appareil.) Toutefois, la meilleure application de ces techniques est la recherche des directions des contraintes principales : les trajectoires des contraintes ont été déterminées pour un certain nombre de cas particuliers.

Cette communication a donné lieu à de nombreuses interventions ayant pour but de rappeler les travaux antérieurs sur ce problème, en particulier ceux de Hast en Suède et de Denkhaus en Afrique du Sud. Hawkes a donné ensuite quelques exemples caractéristiques de l'étude du pivotage de la charge sur un pilier pendant le creusement des chambres voisines et leur élargissement.

SEEBER, Innsbruck (Autriche) :

Pendant les travaux de l'aménagement hydroélectrique du Kaunertal, de nombreux essais in situ ont été exécutés avec le vérin radial de la Trwag (Tiroler Wasserkraftwerke A. G.), notamment pour le calcul du revêtement de la conduite forcée souterraine et de la galerie d'amenée en charge.

De nombreuses courbes effort-déformation ont été obtenues, permettant d’apprécier les différences de comportement entre les calcschistes, les schistes sériciteux et les gneiss cillés, l'anisotropie de ces roches, et l'effet des injections de ciment sur leur déformabilité.

En particulier, on a observé un fluage important des roches lorsque les fissures contiennent des mylonites et des matériaux d'altération. Ce fluage est très largement diminué par l'injection. Au contraire, on note l'absence totale de déformation permanente sur les gneiss massifs.

PAcrer, Salzbourg (Autriche):

Les mesures décrites portent sur une galerie d'es- sai revêtue de béton projeté, dont les déformations sont mesurées en différents profils, soit par des extensomètres formant les côtés d'un octogone régulier inscrit, soit par un palpeur radial mobile fixé au centre du profil, soit par des rosaces d'extensomètres collées sur le béton. Les déformations profondes sont mesurées par des appareils disposés en forages (et utilisables pendant la progression de la perforation grâce à leur communication avec une galerie voisine)

Rocha:

L'auteur commente les résultats de plus de 100 essais de cisaillement in situ avec une surface cisaillée carrée de $70 \mathrm{~cm}$ de côté. Le tableau II donne quelques résultats avec des angles de frottement internes très élevés et des cohésions faibles même pour des roches très saines; ceci traduit donc l'influence des joints. Pour le granite, et suivant son état d'altération (caractérisé par un indice qui reflète la porosité), la cohésion et l'angle de frottement interne varient dans le même sens.

TABLEAU II

Résultats d'essais de cisaillement in situ (Extrait de Rocha)

\begin{tabular}{|c|c|c|}
\hline 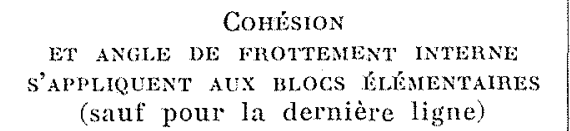 & $\mathrm{kg} / \mathrm{cm}^{2}$ & DEGMẼS \\
\hline 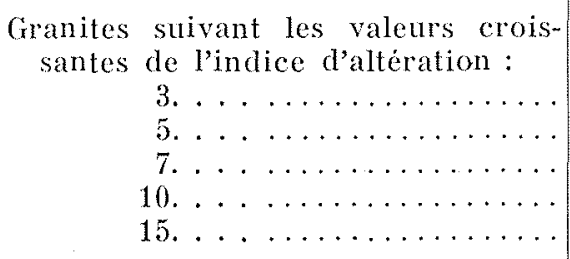 & $\begin{array}{r}13 \\
5 \\
3 \\
2 \\
1\end{array}$ & $\begin{array}{l}62 \\
57 \\
52 \\
46 \\
41\end{array}$ \\
\hline Marnes. . . . . . . . . . . & $\begin{array}{l}2 \\
2 \\
2\end{array}$ & $\begin{array}{l}69 \\
60 \\
63\end{array}$ \\
\hline $\begin{array}{l}\text { Schistes (perpendiculairement à la } \\
\text { foliation)......................... }\end{array}$ & 1 & 70 \\
\hline $\begin{array}{l}\text { Grès (parallèlement à la stratifica- } \\
\quad \text { tion). } \ldots \ldots \ldots \ldots \ldots \ldots \ldots \ldots \ldots\end{array}$ & 1 & 50 \\
\hline Contact grès sur béton . . . . . . . . . & 2 & 63 \\
\hline
\end{tabular}

Dans la discussion, un ingénieur espagnol donne le schéma et les résultats d'essais de cisaillement in situ jusqu'à $4 \times 4 \mathrm{~m}$, exécutés sur des couches de lignite qui séparent des banes calcaires.

M. Müllen insiste sur la valeur comparée des essais en galerie et en sondage; à l'échelle du sondage, on trouve le plus souvent un matériau compact et il faut l'échelle de la galerie pour que les discontinuités caractéristiques du massif rocheux jouent un ròle appréciable.

D'une manière plus générale, le volume intéressé doit ètre à l'échelle de la maille de la fracturation (les essais de Kurobe intéressaient $60 \mathrm{~m}^{3}$ de masif rocheux). 


\section{IV. - Essais au laboratoire}

\section{a) Echantillons}

Plusieurs auteurs se réfèrent à des essais sur échantillons, mais sans allusion aux différences de comportement entre le massif rocheux et l'échantillon, tellement cetle distinction est maintenant acquise. Fumagalli, a comparé les résultats d'essais triaxiaux sur le granite du tunnel du Mont Blanc, suivant la forme des échantillons (cubiques ou cylindriques).

Krsmanovic, Sarajevo (Yougoslavie):

Le matériau naturel discontinu est reconstitué au laboratoire pour des essais de cisaillement. La figure 4 donne les résultats obtenus. En particulier, le rapport de la contrainte tangentielle maximale à la contrainte ultime prend des valeurs caractéristiques : plus grand que 2 dans le calcaire compact, voisin de 2 pour un plan de stratification, égal à 1 pour une mince couche argileuse.
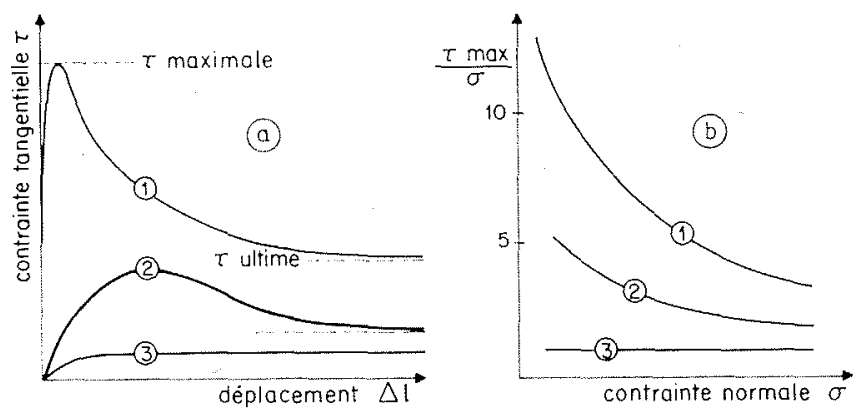

4/ Essais de cisaillement an laboratoire sur blocs rocheux naturels.

1. Calcaire compact;

2. Plan de stratification propre;

3. Mince couche d'argile.

RochA, dans la discussion, s'est montré largement partisan d'essais de laboratoire de ce genre, permettant de faire varier tous les paramètres isolément, ce qu'on ne peut faire in situ. Il propose comme objectif aux chercheurs d'arriver à résoudre au laboratoire tous les problèmes d'un site.

\section{b) Modèles}

Rocha, Krsmanovic, Fumagalli ont étudié des modèles de matériaux formés par empilement de parallélépipèdes avec ou sans imbrication.

Krsmanovic et Milic :

Dans un milieu discontinu à deux dimensions, formé par une seule couche de cubes juxtaposés, la distribution des contraintes mesurées s'éloigne beaucoup du schéma élastique. Sous un plateau chargé, les mouvements relatifs des cubes permettent une concentration des contraintes dans une zone beaucoup plus étroite, avec un maximum à l'intérieur du massif (fig. 5). Lorsqu'on applique à l'ensemble du modèle une précontrainte latérale, limitant le jeu des joints, les contraintes se rapprochent du schéma élastique.

Les mêmes auteurs étudient ensuite la rupture du massif sous la poussée inclinée (d'un barragevoûte par exemple). La figure 6 montre qu'elle s'apparente aux schémas de rupture avec un coin passif et un coin actif.
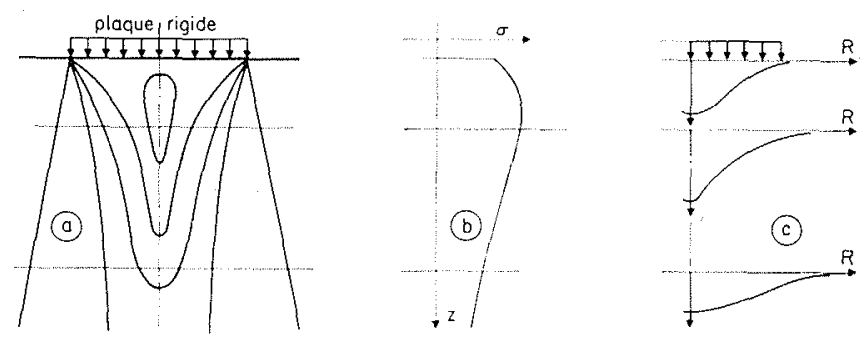

5/ Répartition des contraintes verticales dans un milieu discontinu

(stratifie, a joints imbriqués) sous une plaque rigide:

a) Courbes d'égales contraintes verticales dans un plan vertical;

b) Valeurs des contraintes verticales sur l'axe de symétrie;

c) Valours des contraintes verticales sur 3 plans horizontaux.

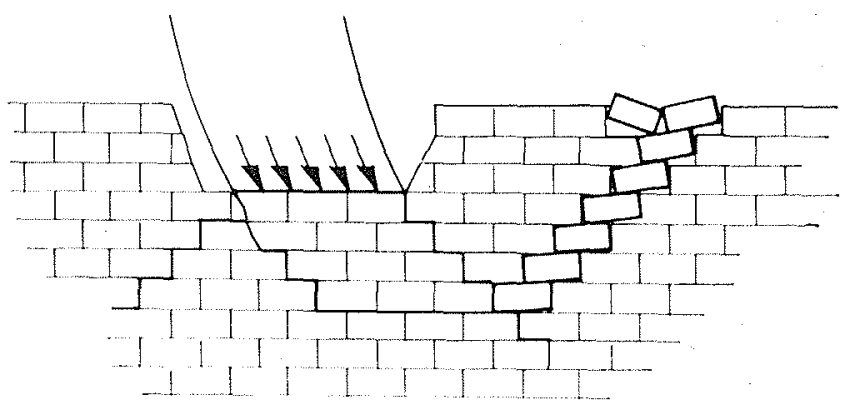

6/ Rupture d'un modele discontinu (stratifié à joints imbriqués) sous une poussée inclinée Con remarque la fissuration très localisée des blocs élementaires)

Fumagalcr projette des photographies d'un modèle du barrage de Vajont avec appuis en matériaux discontinus et essai de rupture. Il donne aussi des précisions sur un modèle de Kurobe où les principales failles avaient été reproduites et où la charge hydrostatique sur le rideau d'injection avait pu ètre ajoutée.

\section{V. - Calcul des ouvrages}

a) Affaissements miniers

HACKETT, Nottingham (Angleterre) :

Dans les mines de charbon d'Angleterre, la théorie de l'élasticité suffit pour rendre compte des principaux mouvements (déformations du mur et du toit, rayon des affaissements de surface). On peut tenir compte d'une certaine anisotropie en ajustant deux coefficients empiriques. Il y a une très bonne correspondance entre le résultat des calculs et les observations effectuées dans un cas particulier.

\section{b) Revêtement des tunnels}

\section{PACHER :}

Lorsqu'un tunnel est revêtu en béton, il y a transfert progressif des contraintes du massif rochenx, et il est important de calculer l'épaisseur du béton en fonction de l'équilibre final. Suivant la durée écoulée avant le bétonnage, cet équilibre est différent et il faut une épaisseur d'autant plus grande que le massif rocheux a évolue davantage.

\section{c) Fondations de barrage}

Srorrc, Sarajevo (Yougoslavie):

On constate, aussi bien sur échantillons que dans 


\section{P. DUFFAUT ET P. LONDE}

le massif, que les concepts de rupture de la mécanique des sols, sur lesquels sont basées les méthodes de calcul utilisées par Meyerhorf et Talobre, ne s'appliquent pas au milieu rocheux discontinu. En particulier, lorsqu'on prend les valeurs extrêmes 0 ou $90^{\circ}$ pour l'angle de frottement interne, ces méthodes ne conduisent pas à des résultats vraisemblables.

\section{ROCHA :}

Les propriétés mécaniques du massif rocheux varient largement d'un point à un autre. L'étude de cette dispersion est très importante, bien que les barrages-voûtes soient très peu sensibles à la présence de zones de faiblesse, même étendues (grâce à leur's facultés d'adaptation).

En l'absence d'essais de cisaillement, il est prudent de calculer le coefficient de sécurité par rapport à une $\operatorname{tg} \varphi$ de 0,6 ou 0,7 . Lorsque tous les essais donnent des valeur's supérieures à 1,4 , on peut prendre ce nombre comme base du calcul. La valeur numérique du coefficient de sécurité dépend très largement des hypothèses. Dans tous les cas, il est recommandé de négliger la cohésion.

\section{VI. - Effet des explosifs}

MüLLER, Salzbourg, (Autriche) :

L'exécution des excavations à l'explosif, à cause de la brutalité des méthodes employées, va souvent à l'encontre du but recherché dans une fouille de barrage. On a maintenant pris conscience du desserrage du massif rocheux autour des tunnels. Dans les deux cas, les schémas de tir conduisent à des économies immédiates, mais il faut ensuite essayer de réparer les dégâts par des injections.

L'effet des explosions sur le massif rocheux est dû essentiellement à la présence des fissures et autres zones de faiblesse :

a) concentration des contraintes à l'extrémité des fissures (l'étude en photoélasticité confirme sur ce point la théorie classique de Griffith, qui assimile les fissures à des ellipses allongées;

b) au passage de l'onde de choc, il y a une perte de frottement temporaire qui permet des mouvements relatifs;

c) l'onde de compression est suivie d'une onde de traction, qui transforme des zones de faiblesse en fissures ouvertes.
Les fissures amortissent l'énergie transmise, notamment grâce à des réflexions, mais ces réflexions produisent des superpositions d'ondes de traction. Lorsque les joints sont remplis d'eau, la transmission est améliorée.

En réalité, les massifs rocheux ont une large possibilité de déformation et on observe souvent des déplacements importants à la surface, sans rupture généralisée. C'est bien dans ce cas qu'on doit penser que la résistance du massif a été largement diminuée.

Une discussion importante a suivi, où les mineur's anglais ont mentionné les études de Trncelin (Ecole des Mines de Paris) et où Bollo a rappelé les résultats d'essais effectués pour Electricité de France dans la galerie du Carla (Tarn).

\section{Conclusions générales}

Il n'est pas besoin d'insister sur l'intérêt d'une telle confrontation entre géologues, praticiens et théoriciens des mines ou du génie civil. Il apparait d'ailleurs un certain équilibre entre des préoccupations purement théoriques et des considérations immédiatement utilisables.

Les différences s'affirment peu à peu entre la mécanique des roches et la mécanique des sols, même si dans quelques cas les mêmes lois restent valables.

1 - On aborde la discussion de la stabilité d'un massif découpé par des discontinuités naturelles;

2 - On s'écarte délibérément des schémas élastiques pour la distribution des contraintes (et on constate, sur des modèles «idéalisés» une concentration de ces contraintes en profondeur);

3 - On commence à chiffrer l'effet des joints sur la résistance du massif rocheux. Leur influence ne devient prépondérante que Iorsqu'on approche de la surface. Les essais sur modèles discontinus et notamment la convergence remarquable des résultats yougoslaves, italiens et portugais ont, à notre point de vue, dominé le colloque. Ainsi prend corps le rêve du projeteur, qui est de prévoir le comportement des massifs rocheux comme il prévoit celui des structures, à partir des résultats de mesures in situ et au laboratoire.

On attendait un peu de nouveaux exemples concrets de description de massifs rocheux (et par exemple la façon d'apprécier le degré de continuité des joints interrompus). Les joints imbriqués, introduits par plusieurs auteurs, sont bien différents des joints interrompus, puisqu'ils ne peuvent progresser par effet d'entaille (ils sont d'ailleurs rarement représentés dans la nature).

On remarquera enfin combien, dans chaque pays et dans chaque profession, les conditions géologiques locales influent sur les préoccupations des chercheurs. Il est tout naturel que l'étude des roches stratifiées soit menée surtout en Yougoslavie et dans les Dolomites. De même, il y a beaucoup de points communs entre les problèmes posés par les travaux hydroélectriques dans les massifs cristallins des Alpes, qu'elles soient françaises ou autrichiennes. 